\title{
Le Train bleu : la couleur et le mouvement d'un voyage
}

Rocío Robles Tardio

\section{CpenEdition}

\section{Journals}

Édition électronique

URL : https://journals.openedition.org/rhcf/516

DOI : 10.4000/rhcf.516

\section{Éditeur}

Rails \& histoire

\section{Édition imprimée}

Date de publication : 1 novembre 2006

Pagination : 131-152

ISSN : 0996-9403

\section{Référence électronique}

Rocío Robles Tardio, «L Le Train bleu : la couleur et le mouvement d'un voyage », Revue d'histoire des chemins de fer [En ligne], 35 | 2006, mis en ligne le 05 avril 2011, consulté le 22 avril 2022. URL : http:// journals.openedition.org/rhcf/516 ; DOI : https://doi.org/10.4000/rhcf.516

Ce document a été généré automatiquement le 22 avril 2022.

Tous droits réservés 


\title{
Le Train bleu : la couleur et le mouvement d'un voyage
}

\author{
Rocío Robles Tardio
}

1 Alors que le XIX siècle touchait à sa fin, les lignes des chemins de fer français s'étendaient toujours davantage, telles des tentacules, avec celles déjà en place. Rails et traverses multipliaient sans cesse leurs kilométrages afin de s'unir avec d'autres en un réseau international qui se formait en Europe par delà les frontières. La nuit n'interrompait pas les voyages. Bien au contraire, ils se paraient alors de couleurs nouvelles plus attirantes encore. Voici donc qu'apparaissent les grands voyages internationaux et intercontinentaux, proposés à une certaine clientèle comme de brefs séjours ou de petites vacances à bord d'un train. À l'idée du divertissement, à celle de la fascination du voyage et du déplacement passif, s'ajoutait celle du plaisir de la nuit, avec les notions de luxe et de confort. Encore une fois, le chemin de fer reflétait et s'adaptait aux exigences « modernolâtres » des classes les plus huppées - la bourgeoisie et l'aristocratie - en les transportant, corps et âme, dans un long voyage à travers les nuits et les jours. En effet, le train de nuit concentrait la commodité et le luxe propres aux grands hôtels tout en garantissant la tranquillité et la sécurité à ses voyageurs. Depuis 1835 , les mots « économie » et " argent »- synonymes de capital - avaient mis les trains en branle à la surface du globe. Ces mots magiques fonctionnaient aussi comme les moteurs et la raison d'être des trains de nuit: "Un voyage ne doit pas empiéter sur vos heures d'activité. Voyagez la nuit en Wagons-Lits. Grâce aux WagonsLits, vous ferez une économie de temps, de soucis, d'argent ${ }^{1}$. » De la sorte, et ce fut l'une des premières conséquences de l'existence de ces trains de luxe des grandes lignes, le chemin de fer cessa d'être le moyen de transport des masses populaires. Luxe et confort redevenaient des privilèges, à reconquérir comme autant de droits démocratiques. À travers toute l'Europe et dans le monde entier, ces trains avaient réussi à mettre sur les rails les luxueux appartements et les hôtels de Paris.

2 Si la devise de ces années-là est « Voyagez la nuit », c'est parce qu'au bout de la nuit les chemins de fer finissent par se dématérialiser, de la même façon que, au milieu du xix ${ }^{e}$ siècle, l'on croyait que les trains pouvaient disparaître engloutis par les tunnels dans 
leur traversée des montagnes. En dévorant les réalisations qui la défiaient, avec leurs auteurs à l'intérieur, c'était comme si la Nature infligeait un châtiment aux hommes pour l'avoir défiée avec leurs grands travaux d'ingénierie, prétendant rivaliser avec la puissance créatrice, et destructrice, jusqu'alors réservée à Dieu. Le phénomène artistique, social et culturel suscité par les trains des grandes lignes pendant le premier quart $\mathrm{du} \mathrm{xx}^{\mathrm{e}}$ siècle, en Europe aussi bien qu'en Amérique, trouve une parfaite illustration dans les épisodes nocturnes du voyage dans le Train bleu.

3 La «Côte d'Azur » est une formule magique utilisée pour la première fois en 1887, par l'écrivain et député Stephen Liégeard, qui donne ce titre à un ouvrage fondateur. Sous cette appellation, on lança une façon de vivre caractérisée par le train et par les vacanciers d'hiver aisés dont la maxime semblait être : investir de l'argent et s'investir dans de nouvelles valeurs! Dans ces années-là, les habitués du trajet entre Calais et la Méditerranée savaient pertinemment que la fête commençait à l'instant même où le majestueux train quittait la non moins remarquable gare de Lyon, à Paris, pour s'élancer à toute allure vers la mer. La fête pouvait même commencer auparavant, autour des tables raffinées du buffet de cette gare, restaurant qui porte aujourd'hui le nom azuréen du train qui lui a conféré tant de prestige. L'affiche réalisée par Zenobel pour cette ligne de la Compagnie Paris-Lyon-Méditerranée (PLM), en 1928, après la rénovation de ses wagons-lits, montre clairement que ce trajet était la grande et vertigineuse pirouette qui poussait à leur comble les émotions débridées, débouchées serait plus approprié, par des bouteilles de champagne à la douzaine. Dans ces années où l'on commençait à claironner la modernité de l'avion, les chemins de fer apparaissaient comme le seul moyen de transport à conserver encore la capacité d'éveiller les plus modernes sensations corporelles, à la manière des wagonnets des montagnes russes. C'est ainsi que, lorsque les canons de la Première Guerre mondiale eurent cessé de rugir, le désir de s'amuser regagna les cœurs des riches entrepreneurs, des snobs qui soutenaient les soirées intellectuelles de la capitale française et de leurs habitués, revenant sous les pinceaux des artistes, les partitions des musiciens et la plume des écrivains.

4 La joie débridée et le luxe revinrent dans la capitale française, emportant les Parisiens sur le même rythme que celui sur lequel la guerre les avait menés dans les tranchées quelques années auparavant. Après les dégâts causés par la guerre, les gens chics voulaient retrouver le calme et profiter des beaux jours sous le soleil. Les corps masculins allaient abandonner les uniformes militaires pour de pratiques tenues de golf et de tennis. Pour leur part, les femmes allaient apprécier la liberté de leurs mouvements sous d'amples jupes blanches en tricot. Tous ces plaisirs, signes de la nouvelle vie moderne, pratique, élégante et entièrement dévolue au loisir, leur furent révélés au bout du voyage le plus festif de tous les temps, celui que leur proposait le Train bleu, faisant par là même entrer ces voyageurs dans la légende ${ }^{2}$.

5 Le 9 décembre 1922, la Compagnie internationale des Wagons-Lits (CIWL) mettait en circulation le premier train de luxe qui reliait l'Angleterre avec le continent en direction de la Méditerranée. Il s'agissait de la ligne Calais-Méditerranée Express, connue presque aussitôt par tous ses habitués sous le nom de Train bleu, même si cette appellation ne deviendra officielle qu'à partir des années trente. La famille Rothschild, qui participait au capital des plus importantes compagnies européennes depuis leur création, entra dans ce projet aux côtés de Lord Dalziel et de René Nagelmackers, tous deux représentants de la compagnie et considérés comme les véritables promoteurs 
économiques et les patrons de fait de ce train aux luxueux wagons-lits en service sur le tronçon Paris-Menton depuis 1877. Mais c'est au directeur de la Compagnie des ballets russes, Serge Diaghilev, et à toute sa troupe de danseurs, peintres décorateurs, costumiers et musiciens, que l'on doit sans doute véritablement la révélation de ce que signifiait le Train Bleu avec leur mise en scène, en 1924, d'un ballet tout à fait particulier ainsi intitulé.

Dans la joie des années vingt, on a baptisé les trains avec des noms de couleurs. «Le train de la Méditerranée » est l'exemple d'un phénomène assez répandu dans l'entredeux-guerres. Il s'agissait alors d'établir un nouvel ordre mondial et les questions artistiques y concouraient sur un pied d'égalité avec les affaires économiques et politiques. Les artistes, main dans la main avec les sciences, la politique et l'économie, ont commencé à peindre les trains en monochromes. Le Train «bleu» en était un exemple, comme le train russe "Étoile rouge ", chargé de parcourir sans s'arrêter l'immense espace soviétique depuis le triomphe de la révolution bolchevique en octobre 1917. Il en fut de même avec la nuance du « wagon vert », destinée à devenir la couleur officielle des trains de la Société nationale des chemins de fer français (SNCF) à partir de 1938, avec l'unification de toutes les compagnies ferroviaires françaises. Par ailleurs, en Italie, c'est dans les années trente que les «trains populaires » firent leur apparition. Sans teinte encore spécifique, ils prirent la couleur et l'odeur des centaines de personnes qui attendaient entassées sur les quais des gares, la promesse politique du " voyage à portée de tous » devenant ainsi réalité. Ce slogan et cette image annonçaient sans doute la mise en circulation des trains allemands en route vers les camps de concentration : le gris glacé du bois des wagons de marchandises va se confondre avec celui des rayures des vêtements des prisonniers, avec aussi la couleur de la fumée des cheminées et des cendres que rejetaient les chambres à gaz et les fours crématoires.

7 La nouvelle aristocratie des années vingt, les familles les plus argentées et même quelques artistes, les plus renommés, étaient prêts à dépenser des fortunes dans les casinos, à collectionner des œuvres d'art, à organiser des soirées littéraires, à mettre en scène des pièces de théâtre et à subventionner la réalisation de films. C'est ce qu'ont fait Colette, Peggy Guggenheim ou les vicomtes de Noailles, qui ont financé eux-mêmes le deuxième film de Luis Buñel - L'Âge d'or (1928), - et le premier de Jean Cocteau, - Le Sang d'un poète (1930) -. Ils avaient en commun un immense désir de changement et sont donc devenus les moteurs et les accélérateurs des mutations les plus radicales de l'époque, dans la mode, les mœurs, les goûts, les loisirs, les boissons et cocktails, les aliments, ou dans ce qui avait trait aux artistes, aux voitures, aux sports... et aussi aux destinations de leurs voyages. Il suffisait de trouver le maître de cérémonie idoine et de s'entourer des artistes et autres créateurs capables de mettre en œuvre le spectacle de ce renouvellement en marche. Car lorsque politiciens et hommes d'affaires s'en furent célébrer la victoire et signer les traités de paix à l'issue de la Grande Guerre, ils s'étaient rendu compte que leurs habits de cérémonie, tout comme leurs comportements, appartenaient à une autre époque, désormais révolue. Cette révélation arriva précisément à l'instant même où le Train bleu faisait sa majestueuse apparition dans la vie parisienne, en gare de Lyon. Des Américains et des Européens riches et aisés désiraient passer l'hiver comme s'il s'agissait d'un nouvel été et, pour ce faire, ils allaient d'abord changer leurs noirs costumes de croque-morts par d'autres aux tissus et couleurs plus dignes d'activités moins lugubres. 
8 À nouvelle conception du voyage, vêtements nouveaux, et nouveaux bagages pour les transporter. La prestigieuse maison Louis Vuitton était là pour ça. Pour elle, une valise équivalait à un wagon. En 1896, le fils de l'inventeur de la valise moderne et initiateur de cette saga familiale, Georges Vuitton, créa un des anagrammes et un des slogans les plus puissants pour l'invitation au voyage. Il utilisa les initiales de son père - L et V pour sigler son négoce, l'empire du malletier : Luxe et Voyage. Il y ajoutait en même temps cette déclaration de principe, toute la philosophie de l'entreprise artisanale: l'art de confectionner une valise, c'est « l'art de traverser le temps ». Rares étaient ceux qui imaginaient qu'une simple valise pouvait révolutionner les mœurs de la société française sous la Troisième République. Ces bagages étaient bien davantage qu'un simple conteneur où placer vêtements et objets d'usage quotidien. En eux-mêmes, ils équivalaient à un voyage et à un moyen de transport moderne, comme le chemin de fer, qui se modernisait au fur et à mesure qu'augmentaient sa vitesse et le confort dont jouissaient les voyageurs. Dans ce sens, le poète Blaise Cendrars, cet infatigable voyageur, n'ignorait pas que le voyage commence dès l'instant où l'on va chercher sa valise, lorsque l'on va acheter ses bagages où vont être accumulés les souvenirs et les trésors que le voyage procurera. Ainsi, Cendrars écrivait dans ses Feuilles de route (1924) la note suivante : «PETITS ACCESSOIRES À LA VIE MODERNE. Le tour du monde d'un voyage de noces. Partir... c'est aller chez Vuitton ${ }^{3}$.» Les valises de cette marque n'étaient donc pas des bagages comme les autres, elles étaient fabriquées pour voyager et devaient se plier aux situations et aux lieux les plus divers. C'est précisément en 1924 que le modèle Keepall de la maison Vuitton apparut, premier bagage souple vraiment moderne, capable de s'adapter à toutes les aventures.

Ces années-là étaient des «Années folles » parce que ceux qui les vivaient avec cette insouciance les voulaient ainsi. L'écrivain et diplomate Paul Morand confessait avoir vécu les années vingt dans un train, ne se sentant vraiment chez lui qu'au fond d'un wagon grand luxe. Pour lui, cette décennie-là fut le temps des voyages : voyager pour aller déjeuner à "La Treille Muscate », la villa que Colette possédait à Saint-Tropez, ou pour visiter Le Lavandou avec Raymond Radiguet et passer la soirée chez Jean Cocteau, ou bien aller manger un poulet rôti avec Darius Milhaud à « La Garoupe », à Lecques, ou encore accompagner le dessinateur, peintre et cinéaste - et un grand ami aussi de Coco Chanel - Paul Iribe dans les rues de Menton. Ainsi racontées, la vie et les prouesses de Paul Morand pendant les années vingt deviennent le thermomètre le plus fiable pour signaler à quel degré la Côte d'Azur bouillonnait artistiquement et intellectuellement. On pouvait y rencontrer un ami dans chacun de ses villages, tous n'étant qu'à quinze kilomètres les uns des autres et le Train bleu était là pour les relier, comme autant de perles précieuses enfilées sur un collier d'aigues-marines. Les loisirs et les amusements y prirent toutes les nuances du bleu et les voyageurs ne furent pas mal inspirés de donner au train le nom de la côte qu'il longeait lors de ses derniers arrêts. La Côte d'Azur se remplissait de gens élégants, d'une foule cosmopolite et excentrique prête à découvrir les bienfaits de la pratique du sport, d'autant qu'à cette époque presque tous les sports étaient à la mode, ainsi que les jeux de hasard, été comme hiver. D'abord le Train bleu était bleu parce qu'il s'est imprégné des nuances bleutées de l'Atlantique en traversant la Manche ainsi que de celles, cristallines et lumineuses, du bleu des eaux de la Méditerranée. Il était aussi bleu parce que dès le moment où il arrivait à Marseille, et jusqu'à son entrée en gare de Menton, le train épousait l'ondoyant contour de la côte française, la locomotive tirant ses wagons se fondant en un seul tracé avec le bord de mer et les rails. Enfin, il était assurément bleu parce qu'il avait été conçu ainsi : ses 
wagons en acier avaient été peints en bleu, apportant une nette amélioration par rapport aux vieux wagons en bois verni. Ces temps nouveaux concernaient aussi les matériaux de construction. Introduire en 1922 des wagons-lits en acier n'était en rien une nouveauté puisque la même CIWL en avait déjà commandés aux usines Pullman de Chicago en 1913 pour les mettre en service en Europe. Mais une fois la guerre terminée, la provenance de ces wagons ne fut plus américaine mais britannique et allemande. Les wagons type $S$ sortirent des usines que la compagnie possédait à Munich. Ce $S$ ne signifiait pas Spécial, qualificatif qu'un train de luxe comme le Calais-Méditerranée pouvait mériter. Cette lettre indique la matière première avec laquelle ils ont été fabriqués : steel. L'acier avait remplacé le bois. On entendait ainsi construire aussi des maisons, urbaniser des territoires et ériger de nouveaux palais susceptibles de durer, capables de résister à une guerre aussi destructrice que celle qui venait de s'achever. Le danger du bois inflammable disparaîtrait avec le froid acier des nouveaux wagons puisque, s'ils venaient à atteindre une très haute température, ils la verraient baisser en arrivant sur la côte sous l'effet des vagues de la Méditerranée. Dans cette éventualité, l'écume de la mer et les embruns auraient joué le rôle des bulles du champagne servi, et bu, au bar du Train Bleu qui, à en croire la renommée, surpassait en qualité le Ritz.

10 Les matériaux constituent l'une des revendications les plus importantes et les plus insistantes émises par une grande partie des artistes qui avaient lutté dans les tranchées pendant la Première Guerre mondiale et par d'autres témoins des premières lignes de retour dans leurs ateliers. Pour ainsi dire, la majorité de ceux qui avaient pris part au conflit estimaient avoir participé à la destruction totale du continent, et ils étaient décidés à reconstruire la vieille Europe dévastée, à la relever de ses ruines. C'est alors que va apparaitre un nouveau type d'artistes-constructeurs, qu'on crée aussi les ateliers de matériaux et métaux dans les différentes écoles d'arts fondées afin de relancer une nouvelle idée de l'art, de l'artiste et de la production artistique par rapport aux principes mécaniques dominants dans la deuxième ère industrielle dont la base théorique résidait dans la notion d'une nouvelle société technicisée et régie par des lois de l'économie, de la précision, de l'efficacité, de la productivité et de la beauté dérivées du modèle de la machine comme pièce intégrée dans l'usine. On peut énumérer bien des peintres, sculpteurs, affichistes et architectes agissant et s'habillant comme des maçons modernes. "Moins de sentiment, plus de matériaux!", voilà la devise que l'artiste russe El Lissitzky lançait dans ses conférences défendant une culture des matériaux contre la culture de la peinture sentimentale et individuelle ${ }^{4}$. Encore en Russie, à Petrograd, Vladimir Tatlin fut chargé, en 1919, du cours «Étude du volume, matériel et construction", à la VkhUTEMAS, l'École des hautes études artistiques et techniques réorganisée et fondée après la révolution. À Moscou, Alexander Rodchenko était le professeur de l'atelier du métal à la Narkompros, la subdivision artistique-industrielle du Département des arts visuels. En Allemagne, Johannes Itten enseignait aux élèves du Bauhaus de Weimar la couleur et la composition au cours préparatoire destiné aux futurs artistes du monde industriel. Quant au béton, au verre, aux poutres et aux câbles d'acier, ils révolutionnaient les modes de construction avec les solutions qu'ils apportaient aux problèmes de l'espace et à la disposition rationnelle de l'espace dans l'architecture moderne comme l'ont montré par exemple Auguste Perret, Walter Gropius, Ludwig Mies van der Rohe, Robert Mallet-Stevens et Le Corbusier. 
11 Serge Diaghilev, à ce moment-là directeur de la Compagnie des ballets russes, voulait mettre en scène pour la saison de 1924 quelque chose de complètement neuf. Une fois encore, après le remarquable succès de Parade (1917), Jean Cocteau n'allait pas le décevoir quand il lui présenta le projet du Train bleu (fig. 1). Ce ne devait pas être un ballet ordinaire : non seulement parce que sa propre compagnie se caractérisait par la rénovation permanente, tant de ses conceptions scénographiques que de celles de la danse, mais parce qu'il s'agissait d'un pur et complet divertissement. Le Train bleu de Cocteau est une pantomime dansée, un jeu de gymnastique en un seul acte que l'on pouvait confondre avec un tableau musical faisant la publicité des nouvelles façons de vivre acquises par la voie des trains de luxe, puisque sa représentation évoque toutes les nouvelles activités, les loisirs et les plaisirs découverts grâce au Train bleu. Voilà comment ce train suscita les envies de sports et de Côte d'Azur et comment il apprit à jouer au golf aux snobs européens, leur faisant abandonner les habits de Paul Poiret pour les jupes pratiques, les vestes et les pantalons blancs en tricot signés Coco Chanel. Ils réservèrent désormais leurs sombres tenues de soirée pour les fêtes du casino de la Jetée-promenade à Nice, cette construction métallique surgie sur la plage, marchant presque sur la mer telle la prolongation naturelle du chemin de fer. Même si ce paradis du jeu ne se voit pas dans le ballet, le public le connaît fort bien grâce aux annonces, aux affiches et aux réclames publicitaires proposées par la compagnie PLM.

Ce ballet, dont la première eut lieu le 20 juin 1924 au Théâtre des Champs-Élysées à Paris, servait de contrepoint à un programme d'opéra de Mozart. Sa brochure, outre la liste des noms de tous ceux qui avaient contribué au Train bleu, incluait quelques dessins signés par Picasso. Ils représentaient une danseuse dans sa loge en train de remonter ses bas, de se maquiller et de se peigner avant d'entrer en scène. Si l'on y prête attention, les spectateurs pouvaient penser qu'il s'agissait d'une variation du Lac des cygnes, mais une autre surprise les attendait car, dans ce ballet, les danseuses ne portaient pas de tutus blancs. Le Train bleu de Diaghilev-Cocteau apparaît comme l'exemple du ballet des temps du fer et de l'acier. Il montre comment un moyen de transport pouvait influencer et modifier les comportements de l'homme moderne, et ouvre aussi de nouveaux chemins vers le soleil et ses plaisirs.

13 Le Train bleu est une danse comique qui rendait compte de la façon de s'amuser des gens aisés et du rôle que les artistes y jouaient. Nul mieux que Jean Cocteau pour en témoigner. Il était l'un des meilleurs connaisseurs de la Côte d'Azur, l'ami de comtes, de peintres, d'écrivains et de sculpteurs, séjournait à Villefranche et était toujours le bienvenu là où l'on cuisinait, buvait ou respirait cet « esprit bleu » qui émanait de la ligne Calais-Méditerranée. Le ballet fonctionnait aussi comme une sorte de réclame de la Côte, faisant d'elle un lieu de référence pour la création artistique où l'on pouvait poursuivre les soirées littéraires entamées à Paris et prolongées au bar dans le train. Selon Cocteau, l'argument du Train bleu est si simple que son livret ne contenait pas de texte. La musique, composée par Darius Milhaud, maintenait tout du long un rythme drolatique qui traduisait en notes l'allégresse des voyageurs à peine arrivés au bord de la mer. Avant que la pièce ne débute, un air de fanfare écrit exprès par Georges Auric invitait les spectateurs à admirer le rideau, réalisé d'après une gouache de Pablo Picasso - La Course, deux femmes courant sur la plage (1922) (fig. 2). Diaghilev voulait compter une fois de plus sur la participation de Picasso aux décors de ce ballet, mais le peintre de Málaga, las de ses dernières collaborations avec les ballets russes - Parade (1917), El sombrero de tres picos (1921) -, refusa. Toutefois il offrit une gouache au cas où 
celle-ci pourrait s'avérer utile. Ce qui fut le cas. Le prince Shervashidze, ami de Diaghilev, fut chargé de l'agrandir afin d'en faire un grand rideau. Naturellement ce que personne n'imaginait, c'est que le jour de la première, Picasso irait voir le résultat et signerait ce rideau comme son œuvre, en ajoutant au passage une cordiale dédicace à Diaghilev ${ }^{5}$.

Figure 1. Photographie du ballet Le Train Bleu, ca 1924,

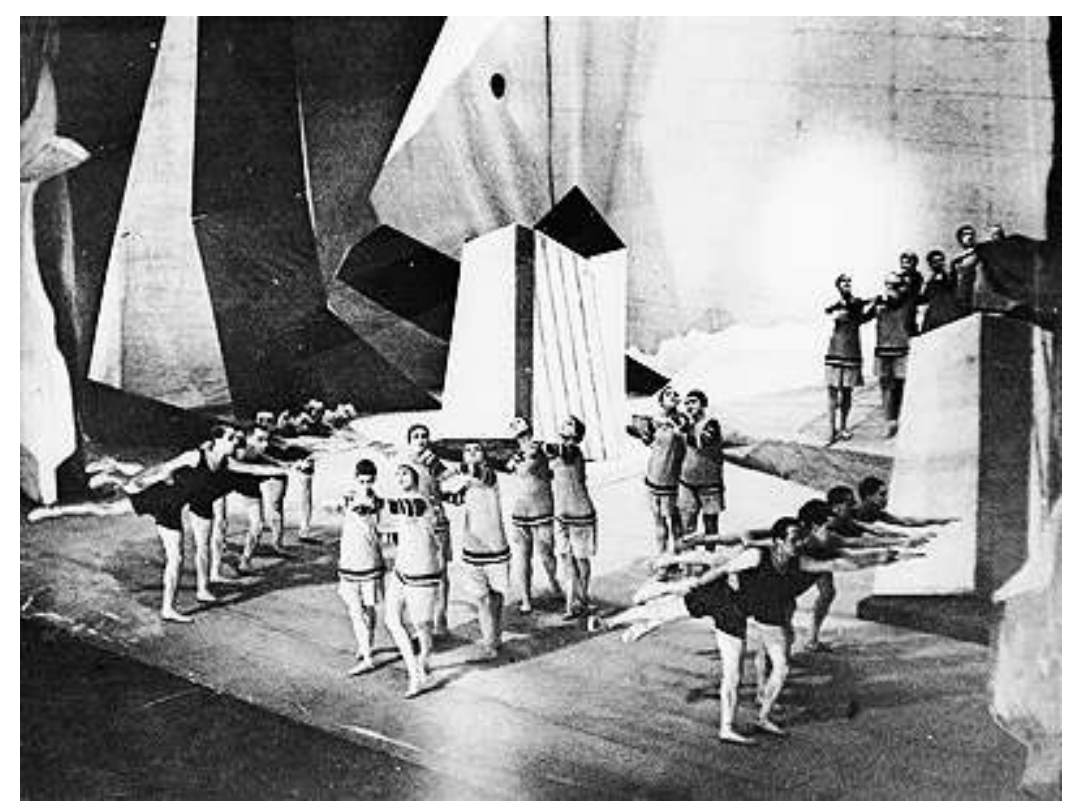

Cl.The Times, d.r.Coll. Bibliothèque-Musée de I'Opéra, album Kochno @ BnF.

Figure 2. Pablo Picasso, La Course, deux femmes courant sur la plage, 1922.

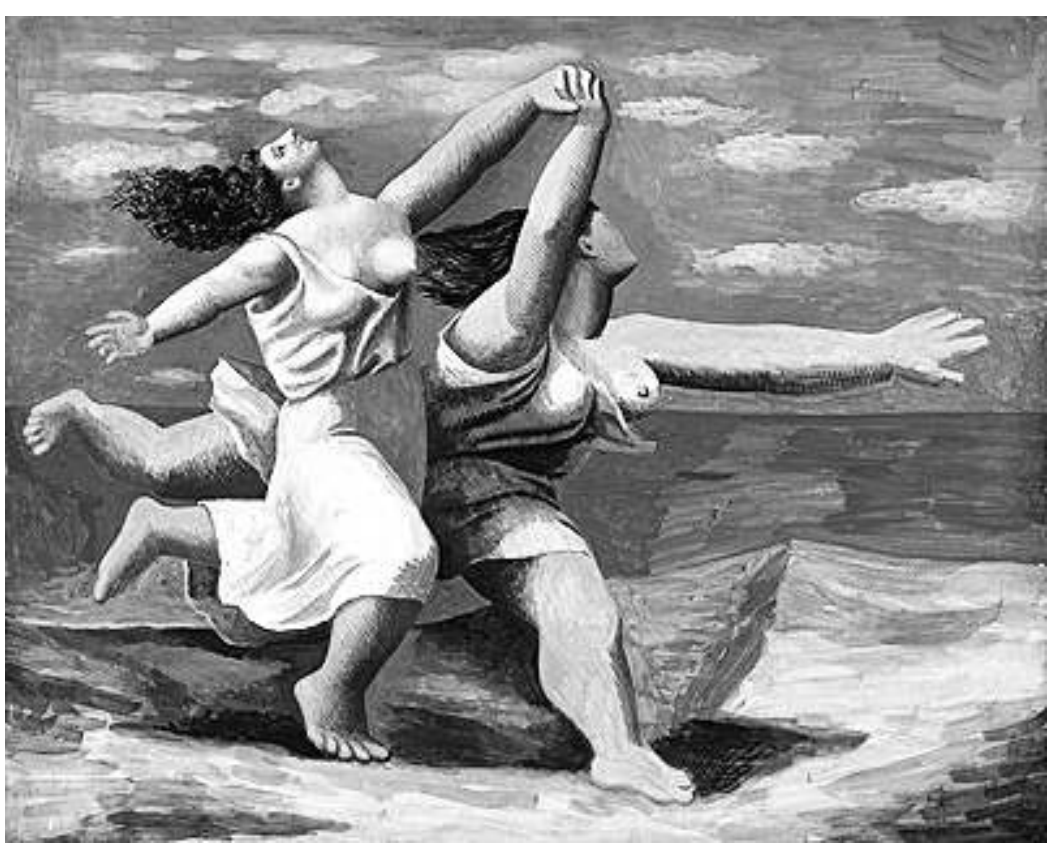

(c) Succession Picasso 2006. Gouache sur contreplaqué (0,325 m x 0,415 m), Paris, Musée Picasso. RMN - Jean-Gilles Berizzi 
14 où le train quitte la gare de Nice et lorsque les voyageurs se retrouvent au bord de la mer $^{6}$. Dès cet instant, ils récréent, toujours grâce à la danse et à des exercices gymniques à la mode, l'activité normale de leur première journée sur la Côte. On peut dire qu'après avoir vu les deux gigantesques femmes de Picasso courant sur la plage, et une fois le rideau levé, les voyageurs, ravis de fouler enfin le sable et la pelouse du terrain de golf au terme de leur voyage de nuit dans le Train bleu, apparaissaient en scène vêtus comme il convenait pour pratiquer n'importe lequel des nouveaux sports. Ces estivants particuliers portaient des vêtements créés gracieusement par Coco Chanel. Ce n'était pas des costumes ordinaires mais des maillots de bain, jupes et sweaters qui procuraient une grande liberté de mouvements, facilitant l'exécution de la chorégraphie conçue par Bronislava Nijinska, la sœur de Nijinski et membre du ballet russe, et la démonstration de la puissante élasticité du danseur Jean Dolin. Cette nouvelle façon de s'habiller allait s'imposer bien vite pour s'ancrer dans la vie quotidienne et s'imposer comme tenue de ville courante (pratique et chic, même dans sa simplicité). Le ballet se révélait être le catalogue et le circuit de diffusion des nouveaux habitus ferroviaires, touristiques et sportifs les plus raffinés, découverts et assimilés par l'élite économique et culturelle. Bref, il n'existait pas meilleure vitrine que ce Train bleu pour montrer à un nombreux public comment il fallait désormais s'habiller si l'on montait dans ce train pour la Côte d'Azur. Les vêtements de Chanel semblent alors faire de la provocation par leur simplicité, par les couleurs choisies - le noir, le blanc et les rayures -, par les coupes et par le genre des tissus (jerseys). Sans oublier l'irrévérence de pousser les femmes à se faire couper les cheveux, et même asymétriquement.

evant cet étalage de modernité dynamique et portée aux excès, il faudrait se poser la question : qu'ont donc exactement vu et compris les spectateurs du Train bleu en 1924 ? Peut-être quelques-uns se sont-ils demandé, à l'issue de la représentation, où était donc ce train qui prêtait son nom au ballet. Les habitués de la Côte d'Azur, eux, se seront sans doute reconnus dans ces gymnastes car ils savaient que, partant de Paris habillé d'une façon, on arrivait à destination vêtu tout autrement. Ils savaient aussi qu'ils ne faisaient pas que voyager de nuit dans un train de luxe. Ils occupaient en même temps un bar luxueusement orné de marqueteries aux murs et aux plafonds signés par René Lalique et René Prou, comme pour siroter un cocktail dans les bars ou les salons de l'aristocratie parisienne, entourés d'artistes et d'écrivains, ou dans les restaurants ouverts sur les Champs-Élysées. Pour d'autres, Le Train bleu pouvait n'être que le nom d'un bar roulant très chic qui restait ouvert toute la nuit, un endroit où prendre un apéritif pendant que la locomotive filait de Paris à Cannes. Quoi qu'il en soit, et dans tous les cas, c'était la vraie conquête du luxe. Le Train bleu avait su prolonger l'activité et le divertissement tout au long du voyage. Même regarder à travers les fenêtres pendant la nuit avait perdu tout intérêt : dehors la nuit était noire. En revanche, ce qui était vraiment intéressant et important, c'était ce qui se passait pendant ces soirées qui se prolongeaient dans le cocon de ces wagons. Et le lendemain matin, de très bonne heure, l'eau de la Méditerranée qui entrait par les fenêtres se chargeait de réveiller et de rafraîchir d'un premier bain de mer les clients de ce bar si particulier . Les vagues qui se brisaient contre les wagons indiquaient que le train avançait parallèlement à la côte et les voyageurs savaient que le moment de changer de tenue était venu pour prendre leur deuxième bain, sous les rayons du soleil celui-là. Au terminus, les wagons

Revue d'histoire des chemins de fer, 35 | 2006 
se transformaient en coulisses et vestiaires où enfiler son maillot car la mer se trouvait juste de l'autre côté de la fenêtre, comme l'a dessiné Roland Hugon dans une affiche pour la SNCF à la fin des années trente (fig. 3).

Figure 3. "Ce soir le train... demain matin Côte d'Azur".

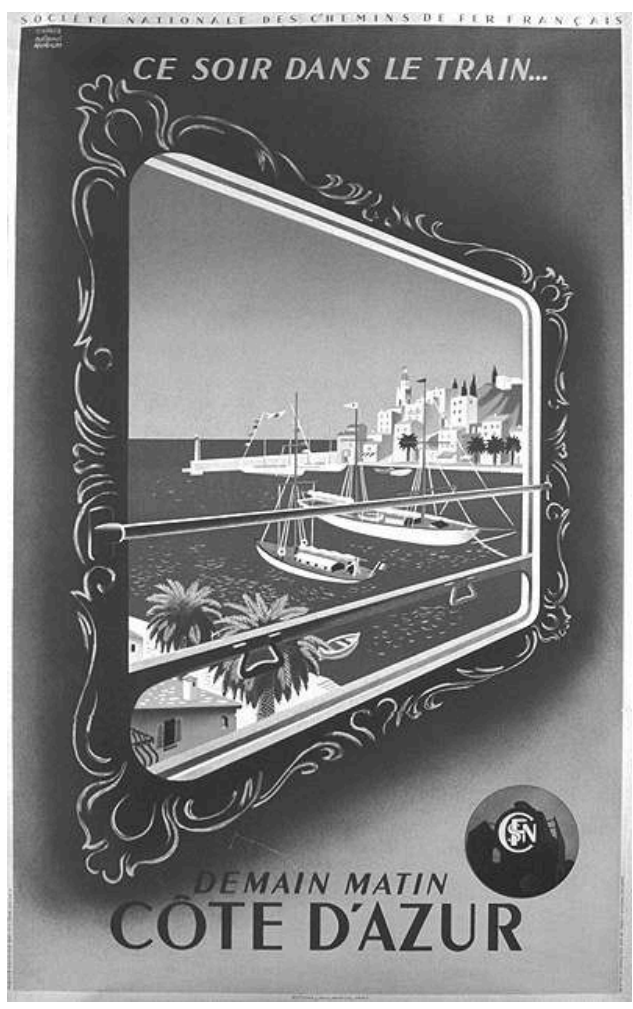

Affiche de Roland Hugon, coll. SNCF. Cl. d.r.

Jean Cocteau a parfaitement synthétisé ce qu'on a vu au théâtre des Champs-Élysées le 20 juin 1924 : il n'y avait pas de train et il n'était pas bleu. Peut-être dictés par le médicament ingéré pour calmer la douleur provoquée par la mort de son compagnon Raymond Radiguet, survenue en décembre 1923, ces mots ont révélé le sens du ballet. Le public a dû aussi percevoir l'aspect insaisissable du voyage. L'esprit était gagné par l'enthousiasme à bord de ce train : cette liberté retrouvée dans les gestes et l'audace des costumes reproduit le rythme des vagues, des tours des roues, de la vapeur de la locomotive et de l'écume des flots. Ce que le public a vu ce soir-là, tout en écoutant la fanfare d'Auric, c'était ça, l'esprit du Train bleu, et l'ivresse qu'il avait déchaînée parmi les voyageurs, en établissant le lien entre leurs corps et la machine à vapeur par le biais des nouveaux sports : le tennis, le golf, la boxe ou la gymnastique. Les baigneuses du tableau de Picasso étaient comme l'incarnation humaine de ces deux trains, l'image de la puissance de la machine. Elles contribuaient aussi par leur course à porter les changements dans les mœurs et les coutumes des gens aisés. Grâce à ces matronesmonstres et grâce à un ballet sans train, les spectateurs ont applaudi à la fin de la représentation, appréciant que personne n'ait osé introduire sur scène la véritable, l'effrayante locomotive du Train bleu. Si les voyageurs modernes avaient acheté de nouvelles valises avant d'entreprendre de nouveaux voyages, c'était une manière de marquer la différence par rapport aux temps du charbon et de la vapeur, conscients qu'ils étaient de vivre dans le luxe au temps de l'électricité hygiénique et abstraite, 
triomphante jusque sur le quai des gares. C'est ce que l'on pouvait lire dans le guide de l'Exposition internationale des arts décoratifs et industriels modernes, tenue à Paris en 1925 : «L'exposition du matériel roulant a permis d'admirer des progrès qui font le plus grand honneur aux Compagnies de chemin de fer, aux techniciens, aux artistes: aujourd'hui le voyage n'offre plus rien de commun avec les pérégrinations de jadis ${ }^{7}$." Le ballet proposé par Cocteau était assurément la confirmation de la grande métamorphose opérée dans la vie quotidienne d'une certaine société européenne moderne, celle des aristocrates, des artistes, des écrivains et des hommes d'affaires au cours des premières décennies $\mathrm{du} \mathrm{xx}^{\mathrm{e}}$ siècle. Le Train bleu est, tel le ver à soie appelé à tisser l'habit de toute la modernité au moyen du subterfuge le plus raffiné qui soit, une mascarade. Et dans ce jeu d'apparences, dans ce voyage vers la simplification et l'abstraction fonctionnelles des formes et des corps, les vêtements de Chanel et les bagages de Vuitton jouent eux aussi un rôle important.

17 Le procédé que la Compagnie des ballets russes avait appliqué à sa brochure a également été utilisé, afin de créer un effet d'attente chez les spectateurs, pour le rideau. Là, il n'y avait pas de danseuses en tutus ni de graciles jeunes filles nimbées de vaporeux voiles blancs. Au contraire, ces deux femmes peintes par Picasso et reproduites par Shervashidze arboraient des péplums agités par le vent à cause de la vitesse de leur course. En d'autres termes, il n'y avait ni gymnastes ni voyageurs du Train bleu. On aurait plutôt dit deux nymphes envoûtées qui, fuyant frénétiquement les griffes d'un Jupiter métamorphosé pour l'occasion en vagues de la Méditerranée, avant que l'écume salée n'effleurât leurs corps, se transformaient en locomotive et wagon du Train bleu. Pour rester dans le domaine de la mythologie, on pourrait également penser que ces femmes sont Charybde et Scylla, les deux nymphes transformées en monstres, gardiennes de chacune des extrémités du détroit de Sicile chargées d'effrayer les navigateurs. En toute hypothèse, le public du théâtre des Champs-Élysées était le témoin d'une version libre et moderne de l'un des plus anciens genres poétiques: les métamorphoses, qu'Ovide lui-même avait définies comme les poèmes qui chantent les formes changées des hommes. Pour la religion des chemins de fer, un rideau comme celui de La Course pouvait passer pour la représentation plastique d'un de ses mythes fondateurs, celui de sa divinité, et marquer une étape de plus vers la définition de son iconographie moderne.

On considère que l'article de l'écrivain russe Osip E. Mandelstam «L'État et le rythme », lui aussi signé en 1924, se fait l'écho de la mode de la gymnastique rythmique, de la pratique du sport en général et de l'école et de la méthode du Suisse Émile JacquesDalcroze en particulier, ainsi que du rôle que doit jouer l'État pour assurer une éducation physique à ses citoyens. Mandelstam insistait surtout sur l'importance et les avantages de la connaissance de toutes les possibilités du corps humain dès l'enfance, en tant que machine de muscles capable de rivaliser avec les machines en acier ${ }^{8}$. Ces années-là représentent le moment de l'apogée des idées et des théories ergonomiques et sportives qui avaient proliféré au cours des premières décennies du siècle et qui avaient comme but principal d'obtenir le rendement physique maximum du corps ainsi que de mettre en valeur la nécessité de s'adapter aux nouvelles circonstances et situations issues de la technicisation progressive du monde, à l'espace du wagon comme de la maison moderne. C'est pourquoi la danse et la gymnastique rythmique étaient utilisées comme autant d'aides et de guides pour savoir dominer le corps en se déplaçant et en occupant l'espace. 
La prolifération de tous ces courants gymnastiques et sportifs au début $\mathrm{du} \mathrm{xx}^{\mathrm{e}}$ siècle se manifeste, avec l'apparition de diverses écoles de danse et d'expression corporelle, comme la réponse scientifique et la systématisation des méthodes de la dite culture physique, laquelle était assumée comme un pendant à la culture spirituelle et intellectuelle. Parmi les rénovateurs de la danse, et après la stèle ornementale aux fameuses arabesques laissée par Loïe Fuller, on remarque l'américaine Isadora Duncan par la simplicité de son vocabulaire gestuel et la récupération du peplon grec comme vêtement de base ${ }^{9}$, et Émile Jacques-Dalcroze, dont la publicité pour sa méthode et son académie à Paris apparaissent dans tous les numéros de l'influente revue L'Esprit nouveau (1918-1925), dirigée par Le Corbusier et Amédée Ozenfant. D’ailleurs, Albert Jeanneret, frère de Le Corbusier et disciple de Jacques-Dalcroze, publia dans cette revue des articles et comptes rendus du concept de la rythmique selon Dalcroze en insistant sur la nécessité pour l'homme moderne de prendre connaissance de son corps, de ses dimensions et de ses possibilités gestuelles et communicatives : «La Rythmique donne à l'individu une connaissance plus fertile de soi-même et fait un individu mieux organisé, mieux armé pour la vie moderne, plus maître de soi ${ }^{10}$.» (Fig. 4 et 5).

Figure 4 et 5 . Photographie illustrant l'annonce du cours de rythmique d'Albert Jeanneret.

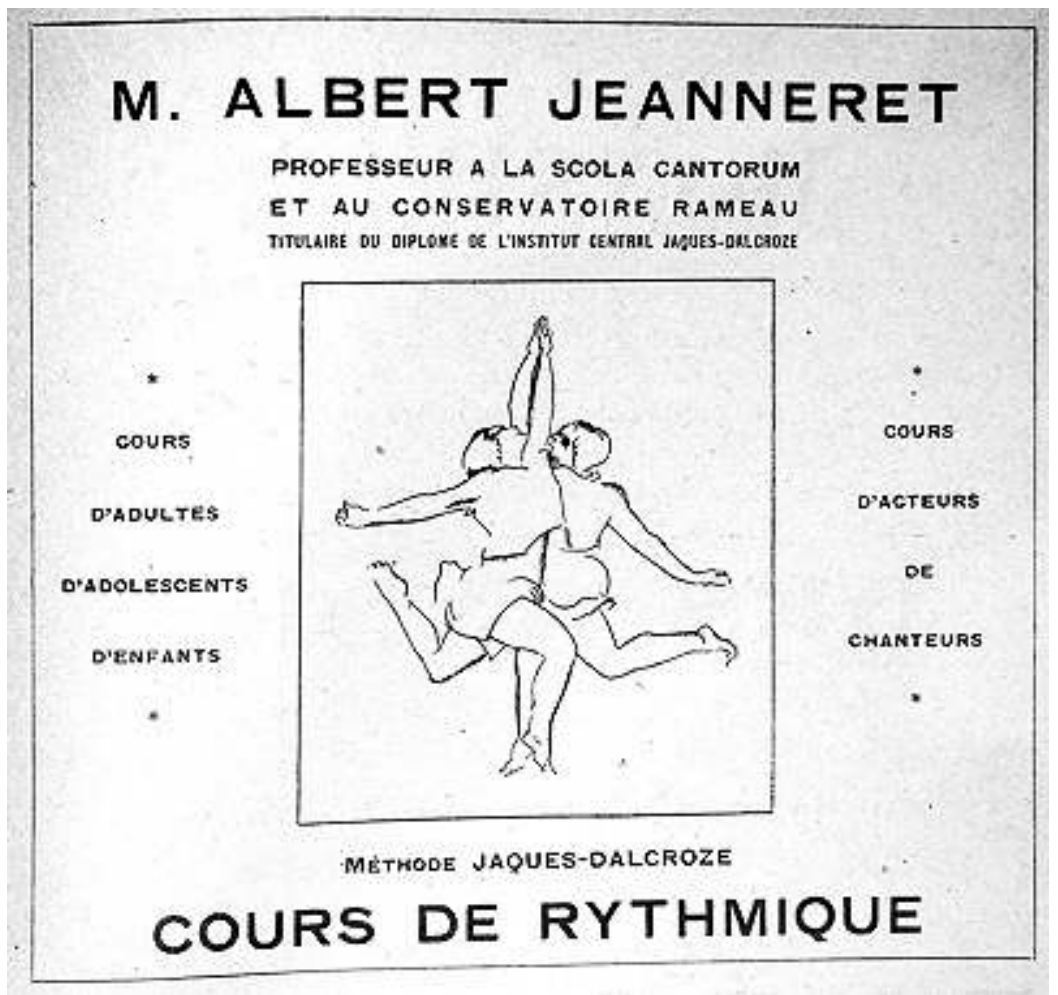




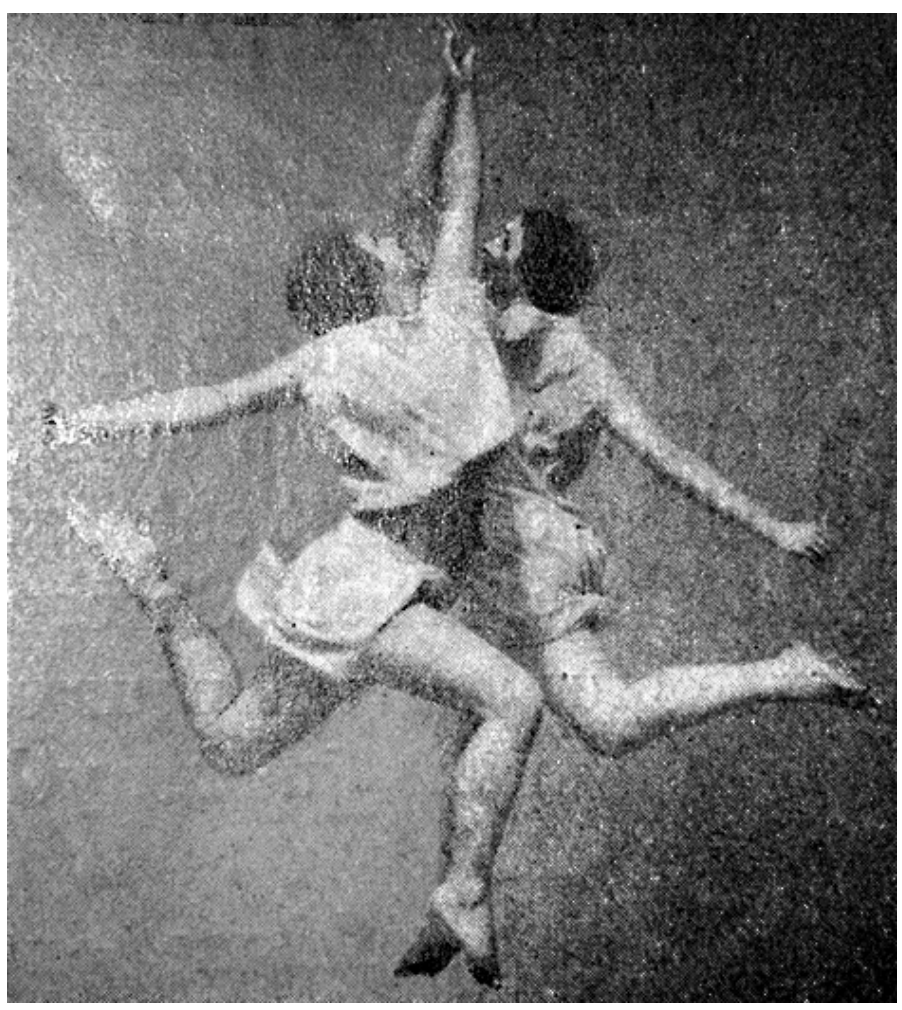

Revue L'Esprit nouveau, n 1, octobre 1921. cl. d.r.

Toutefois, et ce n'est peut-être pas par hasard, les nymphes de Picasso dans La Course ont pris le même chemin que les jeunes filles qui suivaient les cours de cette nouvelle école de gymnastique. Cette similitude gestuelle est mise en évidence au moins sur deux points: l'un visuel et l'autre conceptuel. L'article double signé par Albert Jeanneret dans les numéros 2 et 3 de L'Esprit nouveau - respectivement de novembre et décembre 1920 - sous le titre "La rythmique», était illustré par de nombreuses photographies d'élèves pratiquant les pas et les exercices de la méthode Dalcroze: seules, par deux ou en groupe, tel un chœur de vestales (fig. 6 et 7). C'est dans ses photos que l'on constate l'étonnante appropriation par Picasso, un brin sans gêne - ou est-ce son regard singulier porté sur cette revue en quête de l'iconographie de la vie moderne ? - d'un des pas de cette méthode physiologico-machiniste pour l'interpréter ensuite dans son tableau, parce que ces corps puissants et légers, disciplinés et vêtus à la grecque sont comme des reflets des deux femmes courant au bord de la mer. Nymphes ou machines, nymphes et machines: ces photographies révèlent l'avant annonçant l'après, la puissance et l'acte platonique, la pure métamorphose et le moment précis où l'on peut pressentir le corps et sa transformation, l'anatomie humaine et la locomotive ${ }^{11}$. 
Figures 6 et 7. Photographies parues dans la revue L'Esprit nouveau, n 3, décembre1921.
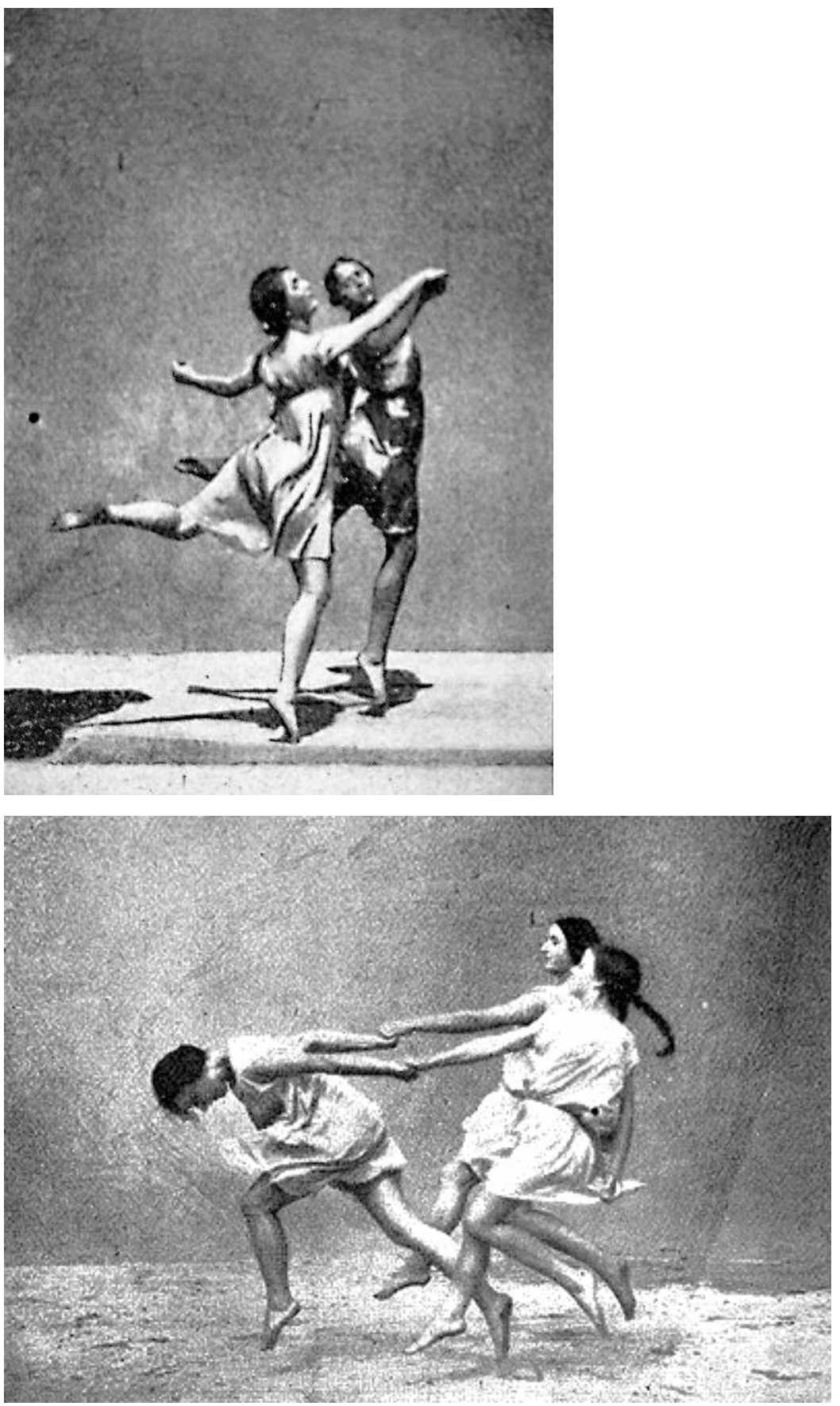

On savait et on soutenait que le but de toutes ces méthodes visait à réussir la synchronisation du corps humain avec le reste des machines afin de le rendre aussi efficace que ses modèles. Pour y parvenir, Albert Jeanneret défendait qu'il était rigoureusement nécessaire de s'exercer à la gymnastique depuis l'âge le plus tendre " pour faire sentir à l'enfant les rapports entre ses instincts, ses impulsions et l'ordre établi dans lequel il se prépare à entrer $\aleph^{12}$. Et voici que tombaient à point nommé les pages de L'Esprit nouveau pour les faire connaître, l'argument du ballet de Cocteau pour les mettre en scène, la musique de Milhaud pour les harmoniser et le double jeu des danseuses et des matrones de Picasso pour démontrer l'équivalence de tous les termes de cette équation de modernité. Le binôme machine-culture grecque devient alors un 
lieu commun au cours des années vingt, surtout grâce à des vitrines publicitaires comme L'Esprit nouveau ou la revue spécifiquement sportive La Culture physique, fondée en 1904. Ainsi, la séquence photographique du Parthénon où pose une Delage Grand Sport pour accompagner l'article de 1921 : « Des yeux qui ne voient pas... Les autos » est un jeu visuel que Le Corbusier avait utilisé pour illustrer l'enchaînement logique et naturel de l'évolution de la capacité rationnelle et critique de l'homme.

Dans ce contexte, après ces chaînons graphico-temporels, la comparaison des deux jeunes filles exécutant un pas de danse avec un train express ne devait surprendre aucun lecteur de L'Esprit nouveau ni aucun spectateur du ballet Le Train bleu : « L'homme redemande à son corps la puissance suggestive d'action. Les manifestations plastiques se multiplient, en même temps que les manifestations sportives ${ }^{13}$."

On peut donc dire que le rôle des revues d'art, d'architecture et de théorie artistique ou sportive, tout comme celui de la peinture et des arts scéniques, a été d'offrir d'abord comme des faits désirables, puis possibles et réalisables, le bouleversement et l'interférence dans la vie des moyens de transport ferroviaires, la régulation du travail et la production. Ainsi, de la même façon que les costumes de Coco Chanel et les cabines de bain prises comme décor du ballet par Henry Laurens - simples et irrégulières coulisses destinées à cacher les métamorphoses de la peau et des vêtements des voyageurs modernes -, Jean Cocteau a trouvé quelques années avant la publication de la revue de Le Corbusier et d'Ozenfant quelques ressemblances entre la machine et l'art grec précisément par leur caractère sobre et utilitaire. Il rendit compte de cette comparaison dans son essai Le Coq et l'arlequin. Notes. Autour de la musique (1918) : « Les machines et les bâtisses américaines ressemblent à l'art grec, en ce sens que l'utilité leur confère une sécheresse et une grandeur dépouillées de superflu. Mais ce n'est pas de l'art. Le rôle de l'art consiste à saisir le sens de l'époque et à puiser dans le spectacle de cette sécheresse pratique un antidote contre la beauté de l'inutile qui encourage le superflu ${ }^{14}$.»

Cocteau refusait pour l'art ce sens de l'utilité et de l'austérité ornementale, innées à la machine, car pour lui l'art est encore ce lieu du vertige et de l'exaltation de tous les sens. Ces affirmations prennent place dans la bataille dialectique et esthétique qui faisait encore rage à l'époque sur la question, vieille d'un siècle, de la participation de l'art à l'industrie et à la technique. Cocteau s'inscrit dans cette ligne ouverte par Théophile Gautier en 1845 avec son article "Plastique de la civilisation ", dans lequel l'écrivain pensait l'art comme la rédemption de l'homme pour ses péchés techniques: «Et puis les nouveaux besoins enfantés par la civilisation ont produit une foule de nouveaux objets et de formes imprévues que l'art n'a pas eu le temps d'idéaliser [...] Supposez des hommes écorchés qui se promèneraient tout sanglants dans les rues avec leurs artères noires et leurs veines bleues, leurs chairs rouges, leurs lacis de nerfs et leurs muscles tressaillants, rien ne serait plus horrible. Eh bien, la civilisation, sous le rapport plastique, offre exactement le même spectacle: les os, les leviers nécessaires y sont, mais la chair et la peau manquent, par conséquent la forme est absente [...] Il faut que l'art donne l'épiderme à la civilisation, que le peintre et le sculpteur achèvent l'œuvre du mécanicien. » Ainsi, même dans la deuxième époque machiniste - définition donnée par Le Corbusier - l'art devait devenir l'antidote et le médicament nécessaires pour éradiquer l'excès décoratif atavique du xix ${ }^{e}$ siècle. un travail précis, régulé, contrôlé, anonyme et continu servirent à bien des artistes 
pour copier les caractéristiques propres de ces machines, chemins de fer inclus, et en doter les hommes. Pour démontrer cette fantaisie mécaniciste, les artistes ont pris comme point de départ l'anatomie et les gestes de l'homme en les assimilant à ceux du mouvement continuel des bielles, des bras articulés, des leviers et des aiguillages. Tout cela conflue dans la conception mécanomorphe de l'être humain selon laquelle les hommes et les machines réussissent tous à effectuer la même chorégraphie mécanique. C'est la raison pour laquelle, dans ces années, on observe une surprenante prolifération de spectacles musicaux abstraits dont les personnages sont des machines ou leurs pendants - locomotives camouflées, trains factices, êtres biomécaniques ${ }^{15}$ : Oskar Schlemmer et Le Ballet triadique, au Bauhaus; Fernand Léger et Francis Picabia respectivement avec Relâche et Entr'acte, ainsi qu'un grand nombre de pièces de théâtre que l'on doit aux artistes soviétiques. L'interprétation mythologique du tableau de Picasso trouve ici le fondement de son explication : l'artiste espagnol se faisait l'écho dans ses œuvres de cet air du temps des mouvements et des idées plastiques puristes, mécanicistes et même abstraites proches du purisme défendu par Le Corbusier dans L'Esprit nouveau.

En fin de compte, toutes ces manifestations sont comme la réponse artistique à une opération de plus grande ampleur visant l'hygiène, la purification et l'abstraction formelle avec lesquelles on voulait caractériser la tragicomédie moderne en cette " deuxième ère machiniste ». De là toute cette obstination à nettoyer des artères par où devait circuler la révolution intellectuelle, morale et physique, offrant ainsi une vision organiciste du monde entier dont les rails sont le squelette. D'autres artistes ont montré les machines telles quelles, comme l'italien Fortunato Depero qui créa en 1924 aussi des vêtements en forme de locomotives pour les acteurs de la pièce musicale Anihccam del 3000.

On doit encore à Paul Morand la confirmation des métamorphoses auxquelles le Train bleu conduisait, que l'on considère le train ou la pantomime ou l'un et l'autre, car il a constaté que les vrais snobs s'habillaient avec des costumes de golf ${ }^{16}$. Autrement dit, le train de luxe et le ballet, par la conduite et les attitudes que tous deux exigeaient, réussirent à imposer un vêtement de type sportif, la chemisette à rayures, et une coupe de cheveux courts, à découvrir aussi des endroits nouveaux et des senteurs nouvelles disséminés le long de la côte méditerranéenne. Paul Morand se souvenait du parfum des eucalyptus dont le feuillage épais procurait une ombre profonde et fraîche, en même temps qu'il servait à masquer les villas privées de Cannes le long du chemin qui montait vers le quartier de la Californie hanté plus tard par Picasso entre 1955 et 1961. Ainsi, en l'an de grâce 1924, l'on découvrit que le paradis artistique pouvait bien se trouver, pour la première moitié du $\mathrm{xx}^{\mathrm{e}}$ siècle, sur les bords de la Côte d'Azur. Et le Train bleu devint sa meilleure publicité. Au cours des décennies suivantes, les artistes se sont établis peu à peu là où le train les portait: Francis Picabia à Martigues, Fernand Léger à Biot, Picasso choisit Antibes, Juan-les-Pins, Cannes et Vallauris. Matisse opta pour Nice, Aimé Maeght et Jean Gabriel Domergue pour Cannes en 1926 et 1927, Auguste Renoir choisit également Cagnes-sur-Mer pour y passer les dernières années de sa vie. 


\section{NOTES}

1. - Annonce parue dans Compagnie Internationale des Wagons-Lits et des Grands Express Européens. Guide. Paris. Service d'été, 27 mai 1962, p. 260.

2. - Pour ce qui est de la notion de loisir dans son rapport au vêtement, voir Thorstein Veblen, Théorie de la classe de loisir (The theory of the leisure class, 1899), Paris, Gallimard, 1970, en particulier son chapitre 7: "L'habillement comme expression de la culture pécuniaire", p. 110-123.

3. - Blaise Cendrars, Feuilles de route. I. Le Formose (Paris, Au sans Pareil, 1924), dans : Du monde entier au cœur du monde, Paris, Denoël, 1987, p. 35.

4. - Citation dans le catalogue de l'exposition: Kasimir Malevich e le sacre icône russe. Avanguardi e tradizioni, Milan, Electa, 2000, p. 45.

5. - D’après Edmonde Charles-Roux, Le Temps de Chanel, Paris, Chêne/Grasset, 1979, p. 197.

6. - Ibid., p. 186.

7. - Exposition internationale des arts décoratifs et industriels modernes. Paris 1925. Rapport Général. Section artistique et technique. VIII. Jouets, Appareils scientifiques, Instruments de musique. Moyens de transports. Classes 16 à 19, Paris, Librairie Larousse, 1928, p. 79.

8. - Osip E. Mandelstam, Sobre la naturaleza de la palabra y otros ensayos, Madrid, Ediciones Árdora, 2005, p. 39 (pas de traduction française sous ce titre).

9. - Voir le catalogue de l'exposition El Teatro de los pintores en la Europa de las vanguardias, Madrid, Museo Nacional Centro de Arte Reina Sofia, Aldeasa, 2000, p. 38.

10. - Albert Jeanneret, publicité pour le «Cours de rythmique », L’Esprit nouveau no 1 (octobre 1920).

11. - Albert Jeanneret, « La Rythmique », L'Esprit nouveau, nº 2 (novembre 1920), p. 183.

12. - Ibid., p. 185.

13. -Ibid. p. 183

14. - Jean Cocteau, Romans, Poésies, E Euvres diverses, Paris, Le Livre de poche, coll. « Classiques modernes », 1995, p. 439.

15. - El Teatro de los pintores..., catalogue cité, p. 47.

16. - Jean des Cars et Jean-Paul Caracalla, Le Train Bleu et les grands express de la Riviera, Paris, Denoël, 1988, p. 68.

\section{AUTEUR}

\section{ROCÍO ROBLES TARDIO}

Doctorante en histoire de l'art, Universidad Complutense de Madrid, Espagne 